\title{
De l'Antiquité à la science-fiction : la réinvention de Babylone dans les représentations artistiques occidentales des $\mathrm{XX}^{\mathrm{e}}$ et $\mathrm{XXI}^{\mathrm{e}}$ siècles
}

From antiquity to science fiction: the reinvention of Babylon in Western artistic representations of the twentieth and twenty-first centuries

\section{Ariane Aujoulat}

\section{(C) OpenEdition} Journals

Édition électronique

URL : http://journals.openedition.org/cel/323

DOI : $10.4000 /$ cel.323

ISSN : 2262-208X

Éditeur

École du Louvre

Référence électronique

Ariane Aujoulat, «De l'Antiquité à la science-fiction : la réinvention de Babylone dans les représentations artistiques occidentales des $x x^{\mathrm{e}}$ et $x \times l^{\mathrm{e}}$ siècles », Les Cahiers de l'École du Louvre [En ligne], 6 | 2015, mis en ligne le 01 avril 2015, consulté le 17 septembre 2019. URL : http:// journals.openedition.org/cel/323; DOI : 10.4000/cel.323

\section{(c) (†) $\odot$}

Les Cahiers de l'École du Louvre sont mis à disposition selon les termes de la licence Creative Commons Attribution - Pas d'Utilisation Commerciale - Pas de Modification 4.0 International. 


\section{De I'Antiquité à la science-fiction : la réinvention de Babylone dans les représentations artistiques occidentales des $\mathrm{Xx}^{\mathrm{e}}$ et $\mathrm{xX \textrm {I } ^ { \mathrm { e } }}$ siècles}

\section{Ariane Aujoulat}

\section{L'iconographie d'un mythe}

L'évolution étonnante des discours plastiques sur Babel et Babylone aux $\mathrm{xx}^{\mathrm{e}}$ et $\mathrm{XXI}^{\mathrm{e}}$ siècles, ainsi que l'extrême diversité des productions artistiques, donne l'impression que la dimension mythique de ces lieux a subi une perte de sens ${ }^{1}$. Cela tient pour partie au fait que ces représentations témoignent d'une volonté de se libérer des codes artistiques traditionnels ou de jouer avec eux, ainsi que d'une tendance à briser les frontières entre les catégories artistiques établies. L'incohérence apparente qui en découle peut expliquer pourquoi, en dépit de la richesse de l'art moderne et contemporain, les études iconographiques demeurent rares pour les périodes récentes. Il est pourtant possible de mener une réflexion sur ce type de sujet en se souvenant qu'il s'agit avant tout, à l'instar de toute production artistique, d'œuvres de l'esprit humain. C'est donc en associant les dimensions historique, anthropologique et philosophique à une telle étude qu'il est possible de comprendre les mutations profondes conditionnant l'évolution des représentations de Babel et de Babylone.

Ainsi que le résume Roland Barthes : « le rapport qui unit le concept du mythe au sens est essentiellement un rapport de déformation ${ }^{2}$ ». En effet, le mythe est un mode de discours symbolique qui permet de mobiliser des images ou des idées de façon immédiate et qui possède donc une puissante capacité d'évocation. Toutefois, cette vision est susceptible d'évoluer selon le contexte historique ou sociologique, car le mythe témoigne des préoccupations de son temps : lorsque celles-ci changent, le mythe doit changer avec elles ou mourir. Par conséquent, le mythe est omnivore : il se nourrit de tout ce qui peut permettre de l'entretenir. Ainsi que nous le verrons, cela peut conduire à des innovations a priori incongrues comme l'association de Babel au monde des livres.

Le mythe est donc collectif et la société se l'approprie et en adapte le récit afin qu'il coïncide avec ses propres questionnements. Or, quel mythe contemporain plus pertinent que celui de Babylone? Désormais en partie confondue avec Babel, cette ville a exercé une fascination ininterrompue de l'Antiquité à nos jours pour son immensité, son luxe et son triste destin, que les textes anciens attribuent à sa décadence ou sa tyrannie. Ses représentations aux $\mathrm{Xx}^{\mathrm{e}}$ et $\mathrm{XXI}^{\mathrm{e}}$ siècles évoluent en fonction de la lecture contemporaine du mythe qu'elle a généré. Ce dernier renvoie plus que jamais à une réalité contemporaine, cependant ses représentations traditionnelles sont inaptes à évoquer cette réalité nouvelle : il subit alors à travers les productions artistiques dont il est le sujet un profond changement à la fois formel et discursif.

\section{De la ville rêvée à la villle recréée}

Jusqu’à la fin du xIx siècle, l'absence presque totale de connaissances archéologiques sur Babylone contraste avec la profusion d'images que fournissent l'art et la littérature en Occident. Le catalogue de l'exposition Babylone en en donne un panorama très intéressant. Du Moyen Âge à la fin de l'époque moderne l'absence de vestiges architecturaux conduit les artistes à proposer des vues essentiellement imaginaires de la ville aussi bien que de la tour. La plus célèbre

1. L'assimilation de Babylone à Babel dans la perception contemporaine nous conduit à traiter le plus souvent ces deux lieux comme un seul.

2. Roland Barthes, Mythologies, Paris, Seuil, collection «Points Essais », 1957, p. 195.

3. Cat. d'exp. Babylone, sous la direction de Béatrice André-Salvini, Paris, Musée du Louvre, 14 mars - 2 juin 2008, Paris, Hazan / Musée du Louvre éditions, 2008. 
de ces vues est la Tour de Babel de Pieter Bruegel l'Ancien (vers 1563) dont deux versions différentes sont connues, l'une au Kunsthistorisches Museum de Vienne et l'autre au musée Boijmans-van Beuningen de Rotterdam.

Dès le milieu du XIX ${ }^{e}$ siècle, les fouilles menées en Mésopotamie inspirent aux artistes comme Edgar Degas, Georges-Antoine Rochegrosse, James Tissot ou encore František Kupka des représentations nouvelles alimentées par l'archéologie ${ }^{4}$. Le site de Babylone, dont la fouille est entreprise de 1899 à 1917 par des équipes allemandes sous la direction de Robert Koldewey, fait l'objet de dessins et de reconstitutions plus proches de la réalité produits par l'assyriologue Walter Andrae. Paradoxalement, c'est au moment où se font ces premières découvertes que les représentations de Babel et de Babylone se détachent de la vision archéologique si prisée au $\mathrm{XIX}^{\mathrm{e}}$ siècle.

En ce sens, Intolerance, film de D.W. Griffith sorti en 1916, fait figure de transition. Le travail de documentation accompli pour ce film est considérable : on en conserve le carnet préparatoire, rempli de collages issus d'ouvrages d'assyriologie ${ }^{5}$. Ladaptation des motifs assyriens et babyloniens dans les décors renvoie aux pratiques du $\mathrm{XIX}^{\mathrm{e}}$ siècle, qui vit là ses derniers feux. Cependant, en s'extrayant des visions critiques biblique et classique pour s'attacher aux découvertes des archéologues tout en mettant en parallèle Babylone avec le monde contemporain, Griffith ouvre la voie à des développements nouveaux. Après ce film, la plupart des évocations de Babylone et de la Mésopotamie dans leur dimension historique revêtent un aspect que nous qualifions de documentaire, c'est-à-dire qu'elles ne servent qu'à constituer un décor crédible pour des œuvres dont elles ne sont pas le sujet principal - notamment dans le cas de bibles illustrées, de bande dessinées ou de films.

Il est plus rare que Babylone en tant que site antique soit le sujet d'une représentation artistique. Anselm Kiefer est l'un des rares artistes à s'être intéressé de près à la civilisation mésopotamienne et à avoir investi ses connaissances dans son travail. Dans Le Croissant fertile ${ }^{6}$ il représente une ziggurat, temple à étages mésopotamien dont Babel est l'archétype, en utilisant de la terre, évoquant ainsi ce matériau de construction originel par la technique de réalisation. Dans son installation Mésopotamie - la Papesse il fait de la Mésopotamie une bibliothèque de livres en plomb de huit mètres de long, dépositaire d'un savoir sacré et inaccessible ${ }^{8}$. Chez Anselm Kiefer, les aspects du mythe de Babylone et les caractéristiques prêtées à la Mésopotamie se doublent d'une réflexion personnelle et d'une symbolique propre à l'artiste pour constituer une vision originale.

Michael Seymour remarque la chose suivante: "One remarkable aspect of Babylon's survival into the present has been its gradual loss of identity as a specific place" ». En effet, la dimension orientale de Babylone et de Babel s'efface au $\mathrm{xx}^{\mathrm{e}}$ siècle, tout comme leur contexte chronologique. Babel et Babylone fusionnent alors pour donner naissance à une ville-tour ancrée dans le monde occidental. C'est le cas avec Babel Revisited de Julee Holcombe (2004) où la ville est un amoncellement de gratte-ciel et d'immeubles en construction (fig. 1). Pour évoquer Babylone, l'artiste préfere donc recourir à l'imagination plutôt qu'aux données archéologiques susceptibles de déconstruire le mythe et de miner sa capacité d'évocation.

Au contraire, l'une des sources visuelles les plus influentes, la Tour de Babel de Pieter Bruegel l'Ancien, est une vue imaginaire. Ce motif est transformé ou transposé dans un autre univers pictural, comme par exemple la série La Tour de Bruxelles d'Éric De Ville ou le Portrait de Jérôme Jacobs de Cédric Tanguy

4. Sur ce sujet, voir par exemple Élisabeth Fontan et Nicole Chevalier (dir.), De Khorsabad à Paris. La découverte des Assyriens, Paris, Réunion des musées nationaux, 1999.

5. Le carnet est conservé à New York, au Museum of Modern Art (Griffith Archives).

6. Fuvre datée de 2009 (Vienne, Essl Museum).

7. Réalisée entre 1985 et 1989 (Oslo, collection Hans Rasmus Astrup).

8. Voir à ce sujet Daniel Arasse, Anselm Kiefer, Paris, Éditions du Regard, 2007.

9. «Un aspect remarquable de la survivance de Babylone à l'époque actuelle a été sa perte d'identité progressive en tant que lieu spécifique », voir cat. d'exp., Babylon. Myth and reality, sous la direction d'Irving L. Finkel et de Michael J. Seymour, Londres, British Museum, 13 novembre 2008 - 15 mars 2009, Londres, British Museum Press, 2008, p. 203. Traduction de I'auteure. 
$(2005)^{10}$. Dans Babel Revisited, la composition du tableau de Bruegel est reprise dans le montage photographique, jusqu'au petit groupe de constructeurs autour de la grue sur le côté inférieur gauche de l'image. Ce phénomène témoigne de la tendance de l'art contemporain à jouer avec les codes de la tradition picturale et à se les approprier. Cependant la transformation de Babylone dans l'art n'est pas causée simplement par une évolution artistique : elle est le symptôme d'un renversement de la signification du mythe qui accompagne un bouleversement bien plus profond des repères, des préoccupations et des systèmes de valeur et qui est dû à la modernisation de l'environnement et des modes de vie en Occident.
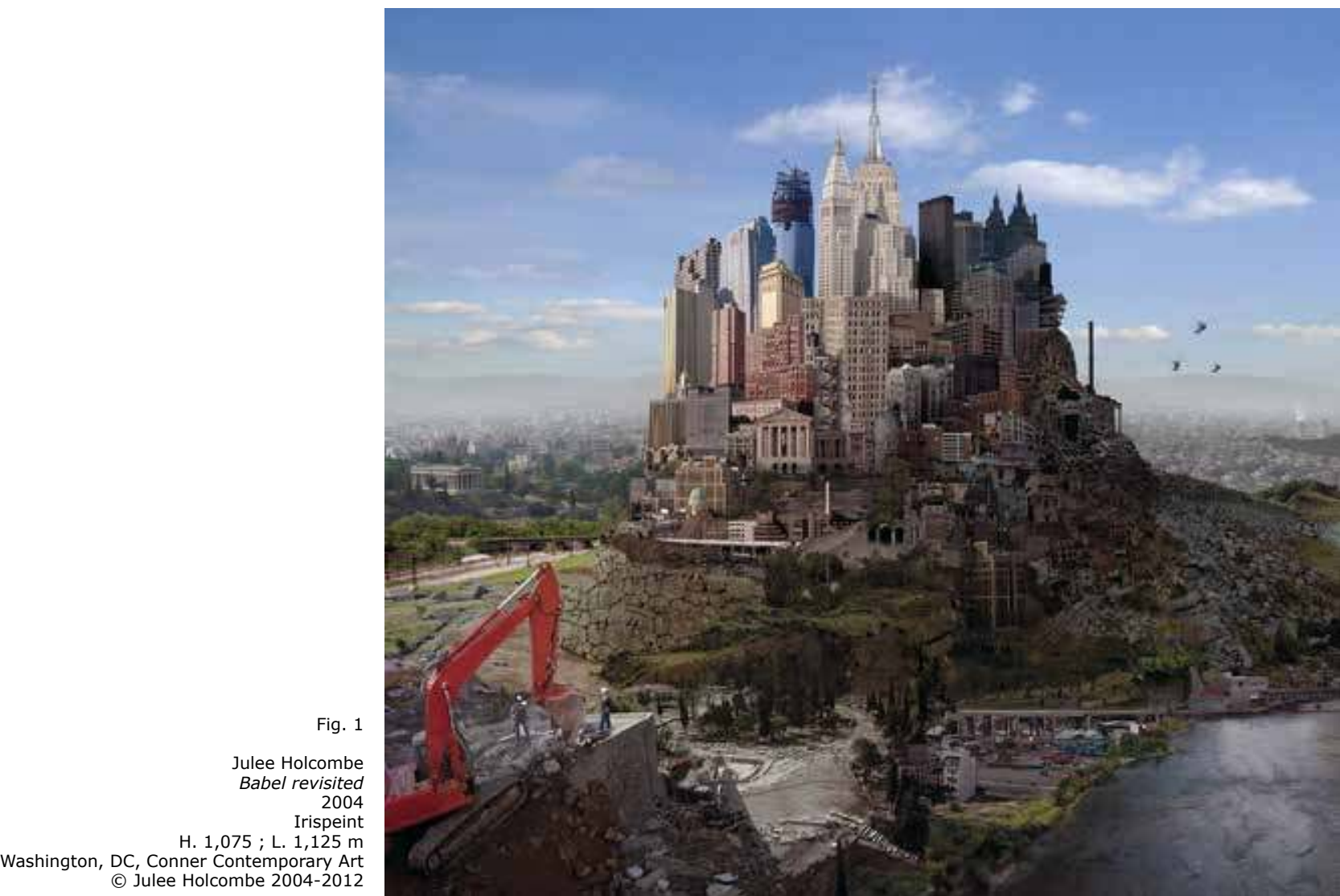

La ville moderne en général fait l'objet, depuis le XIX siècle, d'un amalgame fréquent avec la Babylone biblique, le plus souvent dans la perspective d'une condamnation de la décadence des mœurs. Cette mythification de la grande ville occidentale moderne, étudiée notamment par Roger Caillois ${ }^{11}$, contribue à l'évolution des représentations du mythe, d'autant plus qu'à partir du $\mathrm{xx}^{\mathrm{e}}$ siècle les formes architecturales - tours, gratte-ciel - encouragent l'assimilation avec Babel. Une identification complète s'opère alors, qui peut expliquer l'existence de représentations telles que Babel Revisited.

L'industrialisation est également un facteur de renouvellement des représentations, particulièrement à partir du film Metropolis de Fritz Lang (1927). Dans ce film, le fonctionnement de la ville repose sur des machines actionnées par des ouvriers que l'on pourrait qualifier d'esclaves. Dans une scène où le héros est pris d'hallucinations, ces derniers sont littéralement sacrifiés et dévorés par l'une de ces machines métamorphosée en un démon, Moloch. La méfiance qui se développe à partir des années 1920 à l'égard de l'industrie et des machines trouve ici sa pleine expression ${ }^{12}$. Ainsi le mythe se nourrit des composantes de la modernité : l'expansion des villes, la forme architecturale de la tour et du gratte-ciel, ou encore l'omniprésence de la

10. Voir cat. d'exp. Babel, sous la direction de Régis Cotentin et d'Alain Tapié, Lille, Palais des Beaux-Arts, 8 juin 2012 - 14 janvier 2013, Ennetières-en-Weppes, Invenit, 2012, p. 2, nos 1 à 4 et p. $58, n^{\circ} 42$.

11. Roger Caillois, Le mythe et I'homme, Paris, Gallimard, 1938.

12. Cette vision de la Babel industrielle se retrouve dans l'affiche de l'exposition Le Temps des Gares (Paris, Centre Georges Pompidou, 13 décembre 1978 - 9 avril 1979). 
machine s'y intègrent rapidement. À la faveur de ces assimilations successives, les sociétés occidentales contemporaines s'identifient fortement et très clairement à Babylone pour la première fois dans les représentations. La lecture même du mythe s'en trouve transformée.

\section{Le renversement du mythe}

La plupart des représentations de Babylone et de Babel à partir du $\mathrm{xx}^{\mathrm{e}}$ siècle témoignent d'une nouvelle lecture du mythe qui procède d'un renversement de sa signification. Ce phénomène accompagne une mutation profonde des systèmes de valeurs et des préoccupations qui se produit à la fin de la Grande Guerre. Dans une analyse portant sur cette question, Hubert Bost ${ }^{13}$ met en lumière ce qu'il appelle le renversement axiologique du mythe de Babel. En effet, dans la symbolique traditionnelle, les destinataires du récit s'identifient aux hommes d'après la confusion des langues alors que dans la symbolique moderne, ils s'identifient aux hommes d'avant ou pendant la réalisation de l'entreprise, au moment où ceux-ci parlent tous le même langage. L'histoire de Babel appartenait au passé, elle devient au $\mathrm{XX}^{\mathrm{e}}$ siècle un événement du présent, voire du futur. De même, alors que dans la symbolique traditionnelle l'on déplore la perte de la langue unique cimentant la société, la symbolique moderne fait passer cette langue unique pour le symptôme d'une uniformisation de la société et de l'abdication de la liberté individuelle au profit d'un projet collectif inquiétant. La nouvelle lecture du mythe de Babel qui s'impose au Xx ${ }^{e}$ siècle et conditionne ses représentations lui donne ainsi une forte connotation politique.

Afin de mettre en lumière les implications de cette nouvelle lecture, attardonsnous sur l'aspect linguistique du mythe de Babel: si la question du langage est toujours prégnante dans certaines représentations du mythe de Babel au $\mathrm{xx}^{\mathrm{e}}$ siècle, il présente des développements qui vont plus loin que la simple confusion linguistique. Dès Metropolis de Fritz Lang, l'impossibilité de se comprendre découle non pas de la confusion des langues mais de l'incapacité à communiquer le fond de sa pensée et à dialoguer avec l'autre. L'incompréhension est donc la conséquence d'un problème de fraternité et de cohésion sociale plutôt que d'une différence linguistique. D'autre part, le renversement axiologique du mythe de Babel s'exprime, dans le cas du langage, par la crainte de voir non pas les langues se multiplier, mais s'uniformiser ou disparaitre au profit d'une seule. Cette inquiétude relativement récente contraste avec les nombreuses tentatives qui, jusqu'au début du $\mathrm{Xx}^{\mathrm{e}}$ siècle, consistaient à retrouver une supposée langue originelle ou adamique, mère de toutes les langues. La mise au point de l'espéranto par Ludwik Lejzer Zamenhof en 1887 répondait à l'ambition de proposer une langue internationale qui permettrait de faciliter la communication entre les hommes et d'encourager la fraternité ${ }^{14}$. Au $\mathrm{xx}^{\mathrm{e}}$ siècle au contraire se développent des initiatives de préservation du patrimoine linguistique et de sa diversité, comme la Charte européenne des langues régionales ou minoritaires de 1992. Elle se double d'une critique politique de l'uniformité du langage, qui conduirait à l'éradication de l'individu au profit du groupe. Cette préoccupation est par exemple manifeste dans 1984 de George Orwell (1949) avec la novlangue, langue mise au point dans le but avoué d'uniformiser et de contrôler les pensées.

Ces préoccupations contemporaines assimilant langage unique et dictature ont une forte influence sur la lecture du mythe de Babel qui, comme nous le verrons, est par ailleurs associé à la question de l'esclavage et du totalitarisme. De fait, cela a pu poser des problèmes réels dans le domaine de l'architecture à l'exemple du siège du Parlement européen à Strasbourg, dont les travaux furent achevés en 1992. Il prend la forme d'une tour de plan circulaire à l'aspect inachevé devant laquelle flottent les drapeaux des nations européennes. Le bâtiment, dans sa

13. Hubert Bost, Babel. Du texte au symbole, Genève, Labor et Fides, collection « Le Monde de la Bible », 1985, p. 209.

14. Voir sur ce sujet I'ouvrage d'Umberto Eco, La recherche de la langue parfaite dans la culture européenne, $1^{\text {re }}$ éd. Rome, Edizione Laterza (1993), trad. de I'italien par Jean-Paul Manganaro, Paris, Éditions du Seuil, 1994. 
fonction même, fait inévitablement intervenir la thématique du langage tandis que sa forme architecturale évoque clairement la tour de Babel.

Toutefois, si le mythe est repris visuellement dans l'architecture du Parlement, il est rejeté dans le discours. Il existe une tension entre la volonté de maintenir différents langages dans ce même endroit - c'est-à-dire d'éviter l'uniformisation et le totalitarisme - et celle d'assurer une cohésion et une unité politique, c'est-àdire de ne pas encourager la désunion en exaltant les différences. Ainsi, l'objectif explicite des discours produits autour de ce bâtiment est de "débabéliser le terrain ", jusqu'à renvoyer l'aspect inachevé de la tour au non finito de MichelAnge plutôt qu'à la tour de Babel, dont la référence est pourtant plus évidente ici : "L'image même de la démocratie risquait bien, en effet, d'être la tour de Babel, ce bâtiment où plus personne ne se comprend, ne s'estime, ne s'écoute ${ }^{15}$ ". Le siège du Parlement européen est donc une œuvre problématique : il présente la forme d'une tour de Babel - qui évoque bien sa fonction - alors même que cette forme est vidée de son sens par le discours que l'on choisit d'y apposer. Le mythe de Babel, affirmé visuellement, est donc intellectuellement réfuté. Ce phénomène tient à l'ambiguïté de sa lecture aujourd'hui.

\section{La grande prostituée, éternelle décadente}

La seule caractéristique de la Babylone antique qui demeure constante à l'époque contemporaine est sa nature supposée décadente, notion récurrente dans les textes bibliques et classiques. La figure de la prostituée, incarnation de Babylone, est essentielle pour cet aspect. La prostituée de Babylone peut être convoquée telle quelle ou prendre, dans les arts, la forme d'une femme fatale ou encore, très souvent, d'une danseuse orientale à l'image de Salomé dont les représentations sont nombreuses au XIX ${ }^{\mathrm{e}}$ siècle. À partir du xx ${ }^{\mathrm{e}}$ siècle les beaux-arts ne traitent que rarement de ce thème, en revanche ce personnage est récurrent dès l'entre-deuxguerres dans la littérature, laquelle se fait l'écho des préoccupations de l'époque portant sur la décadence voire la dégénérescence des sociétés occidentales ${ }^{16}$.

Cette figure est très présente au cinéma, notamment dans Metropolis où Fritz Lang fait d'un robot de forme féminine l'incarnation même de la prostituée de Babylone dans une des scènes les plus mémorables du film : à demi nue, elle se livre d'abord à une danse du ventre qu'elle termine juchée sur une bête à sept têtes cornues tandis que s'animent les sept péchés capitaux. Excités par l'apparition, les habitants de Metropolis sont pris d'une fureur destructrice et l'Apocalypse menace la cité. Plus récemment, dans le film Alexandre d'Oliver Stone (2004), les Babyloniennes sont présentées comme des femmes sensuelles et peu farouches qui se mettent à danser lorsqu'Alexandre et ses généraux pénètrent dans le harem. Ainsi, alors que les représentations de Babylone en tant que ville moderne et industrialisée témoignent d'une mutation considérable du mythe, cet aspect féminin hérité du XIX ${ }^{e}$ siècle se retrouve transposé tel quel dans des représentations pourtant très nouvelles par ailleurs. En effet, si les conceptions relatives au mythe de Babylone en général ont beaucoup évolué du fait de l'industrialisation et de l'expansion urbaine ainsi que des nouveaux cadres économiques et politiques, le stéréotype de la femme orientale comme séductrice semble en revanche s'être maintenu dans les mentalités.

\section{Principales nouveautés du mythe introduites dans les représentations}

L'un des aspects de la Babel moderne est l'absence presque totale de Dieu. Les êtres humains réalisent la tour par la seule force de leur volonté et de leur cohésion, en contrepartie ils sont aussi les artisans de leur propre chute. D'après Sylvie

15. Les deux citations sont tirées de Pierre-François Mourier, Le Parlement européen à Strasbourg, Besançon, Les Éditions de I'Imprimeur, 1999, p. 68.

16. Voir par exemple Manhattan Transfer de John Dos Passos (1925), Berlin Alexanderplatz d'Alfred Döblin (1929), Babylone de René Crevel (1929). 
Parizet qui a consacré un ouvrage au mythe de Babel dans la littérature moderne ${ }^{17}$, la vision du mythe est de plus en plus anthropocentrique ou, pourrait-on dire, elle se laïcise. Les constructeurs de Babel, confiants en leurs propres capacités, ne pensent pas à Dieu mais au projet politique qui les motive. Dès lors il n'est pas étonnant que les projets architecturaux liés à Babel ou Babylone revêtent une dimension presque utopiste.

Le Monument à la Troisième Internationale imaginé par Vladimir Tatline en 1919 - mais jamais réalisé - en est un bon exemple (fig. 2) : cette tour-sculpture érigée à la gloire du Komintern, de forme spiralée, devait s'élever à 400 mètres de haut. Elle était composée d'éléments géométriques habitables tournant sur eux-mêmes et intégrait entre autres un réseau de circulation, des projecteurs et une radio de portée internationale prévue pour transmettre au monde entier le message communiste. Repère spatial et politique, elle devait matérialiser l'unité soviétique. Outre ses similitudes formelles avec la tour de Babel, elle fut perçue comme telle par une partie du public. Les projets de la Tour Lumière Cybernétique de Nicolas Schöffer ou de la Nouvelle Babylone de Constant Nieuwenhuys, tous deux imaginés dans les années 1960, participent de la même dynamique. Ironiquement, à l'instar de la tour du mythe, aucun de ces projets babéliens ne fut jamais achevé.
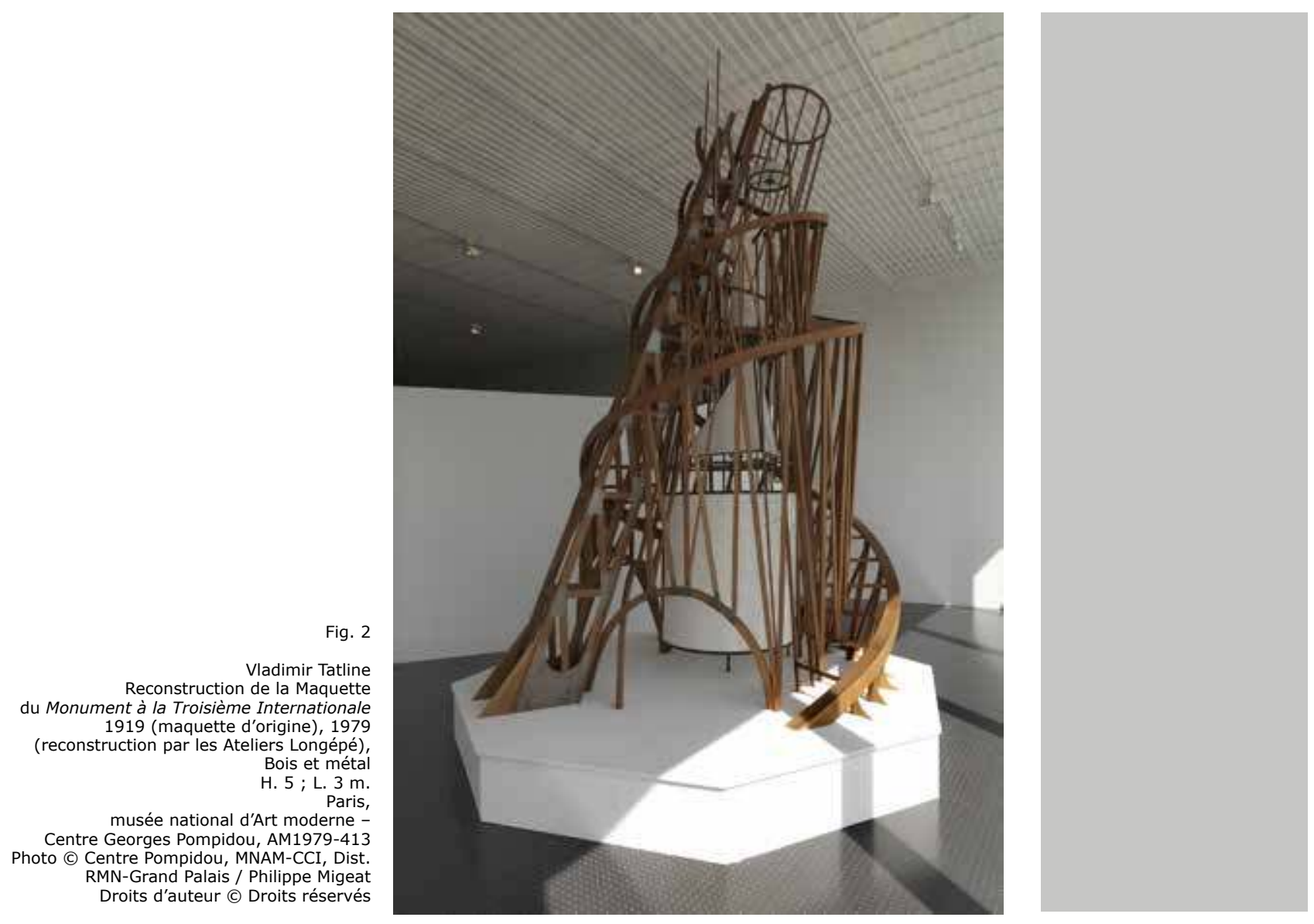

Si l'on excepte les exemples issus de l'architecture, le chantier de Babel devient dans l'art $\mathrm{du} \mathrm{xx}^{\mathrm{e}}$ siècle un lieu d'oppression et d'aliénation dont la vanité n'a d'égale que l'absurdité. Le projet est tellement démesuré qu'il n'est plus appréhendable par l'être humain, lequel finit par y participer sans plus en comprendre le sens ${ }^{18}$. L'absurdité du chantier babélien, qui pourtant engage toute une communauté à travailler jusqu'au bout de ses forces, est le sujet d'une bande

17. Sylvie Parizet, Babel, ordre ou chaos? Nouveaux enjeux du mythe dans les œuvres de la modernité littéraire, Grenoble, ELLUG, collection « Ateliers de I'imaginaire », 2010.

18. Phénomène décrit notamment dans une nouvelle de Franz Kafka, La muraille de Chine, publiée en 1936. 
dessinée de François Schuiten, La Tour $\left(1987^{19}\right)$, dans laquelle un maçon employé pour entretenir l'édifice décide de monter au sommet et, y parvenant au prix d'immenses efforts, se rend compte qu'il n'y a rien en haut. Il est alors confronté à l'inutilité de sa propre existence, ayant consacré toute sa vie à la réalisation d'un projet absurde.

Par ailleurs, sur le chantier de Babel, seule importe la construction de la tour : l'individu ne compte guère et est facilement remplaçable. Aussi n'éprouve-t-on aucun scrupule à l'exploiter : dans Metropolis de Fritz Lang, au cours de la scène racontant le mythe de Babel, les ouvriers du chantier sont présentés comme des esclaves au crâne rasé, marchant tête basse et tirant des blocs de pierre cyclopéens. Parallèlement, dans les scènes portant sur la ville de Metropolis ellemême, les ouvriers chargés d'actionner les machines accomplissent leur tâche avec résignation, les yeux hagards, et sortent de l'usine vidés de leurs forces et de toute volontée ${ }^{20}$. En réalité rien n'est dit des conditions de travail des ouvriers dans la Genèse et cet aspect n'est pas non plus présent dans les représentations picturales traditionnelles : la thématique de l'esclavage sur le chantier de Babel est donc une composante récente du mythe, qui doit sans doute beaucoup aux combats sociopolitiques du temps. Après Metropolis, l'oppression et l'aliénation deviennent des éléments fréquents de la vision moderne du mythe rapidement repris dans la littérature et les arts visuels ${ }^{21}$.

Dans cette perspective, il est intéressant d'évoquer la question du pouvoir à Babylone. Dans l'Antiquité, le souverain de Babylone est souvent perçu comme un tyran orgueilleux tel Nabuchodonosor (Daniel, 4, 26-31) ou efféminé comme Sardanapale (Bibliothèque historique, II, Diodore de Sicile). À partir du xx siècle, ce tyran oriental est remplacé par l'homme d'affaires ou l'ingénieur. Ainsi en va-til, chez Fritz Lang, de Fredersen, le créateur de la ville de Metropolis qui règne à son sommet, ou de Duke Red, concepteur de la ziggurat et auteur d'un coup d'État dans le film d'animation Metropolis de Rintarō (2001). Ces représentations traduisent une conception tout à fait contemporaine du pouvoir résumée par JeanFrançois Lyotard : la classe dirigeante n'est " plus constituée par la classe politique traditionnelle, mais par une couche composite formée de chefs d'entreprises, de hauts fonctionnaires, de dirigeants des grands organismes professionnels $[\ldots]^{22}$ ". La domination politique exercée par le monde de la finance se trouve ainsi très logiquement associée aux représentations babéliennes des $\mathrm{xx}^{\mathrm{e}}$ et $\mathrm{XXI}^{\mathrm{e}}$ siècles. Le caractère négatif du pouvoir babylonien peut prendre des proportions extrêmes : la dimension politique du mythe de Babel sous-tend la structure narrative du film de Fritz Lang du début à la fin et, bien qu'elle soit au service d'une vision utopiste, Siegfried Kracauer en fait un film pré-nazi ${ }^{23}$. Babel est donc très vite assimilée à la dictature et au totalitarisme, particulièrement à partir de la Seconde Guerre mondiale.

Babel et Babylone fusionnent également dans le thème de la chute, aspect essentiel du mythe. Alors que dans la Genèse la tour de Babel est simplement inachevée, beaucoup de visions contemporaines lui associent l'idée d'effondrement. Les premières images de destruction de la tour datent de la Renaissance ${ }^{24}$ alors qu'elles ne sont pas justifiées par le texte biblique. D'autre part, Babylone est vouée dans la Bible, et particulièrement dans l'Apocalypse, à la destruction totale. Par conséquent, les représentations de Babylone et de Babel à partir du $\mathrm{Xx}^{\mathrm{e}}$ siècle prennent des allures de fin du monde. Ainsi, dans les montages de Zhenjun Du comme The Snow (2012), la tour de Babel domine un monde hostile où l'humanité impuissante est en proie aux tourments (fig. 3), tandis que dans le film d'animation Metropolis de Rintarō, la tour de Babel se révèle être une arme nucléaire. Loin d'être l'annonce d'un monde meilleur débarrassé de tout mal,

19. François Schuiten, La Tour, Casterman, 1987.

20. Cette vision se retrouve dans le tome 5 de la bande dessinée Universal War One de Denis Bajram (2005) où Babel symbolise l'effet mortifère du capitalisme et de la mondialisation.

21. Voir notamment le poème de Pierre Emmanuel, Babel, Paris, Desclée de Brouwer, 1951.

22. Jean-François Lyotard, La condition postmoderne, Paris, Les Éditions de Minuit, collection «Critique », 1979 , p. 30.

23. Siegfried Kracauer, De Caligari à Hitler. Une histoire psychologique du cinéma allemand, trad. de I'anglais par Claude B. Levenson, Paris, Flammarion, 1987, p. 180-181.

24. Op. cit. note 3, p. 457. 
l'Apocalypse contemporaine est une fin du monde violente et sans issue heureuse. $\mathrm{Au}$ sein des représentations contemporaines, seules les images générées par le mouvement rastafari - principalement des chansons et des pochettes d'album de musique reggae - renouent avec la vision biblique de l'Apocalypse en réactualisant Babylone comme symbole de l'Occident oppresseur.
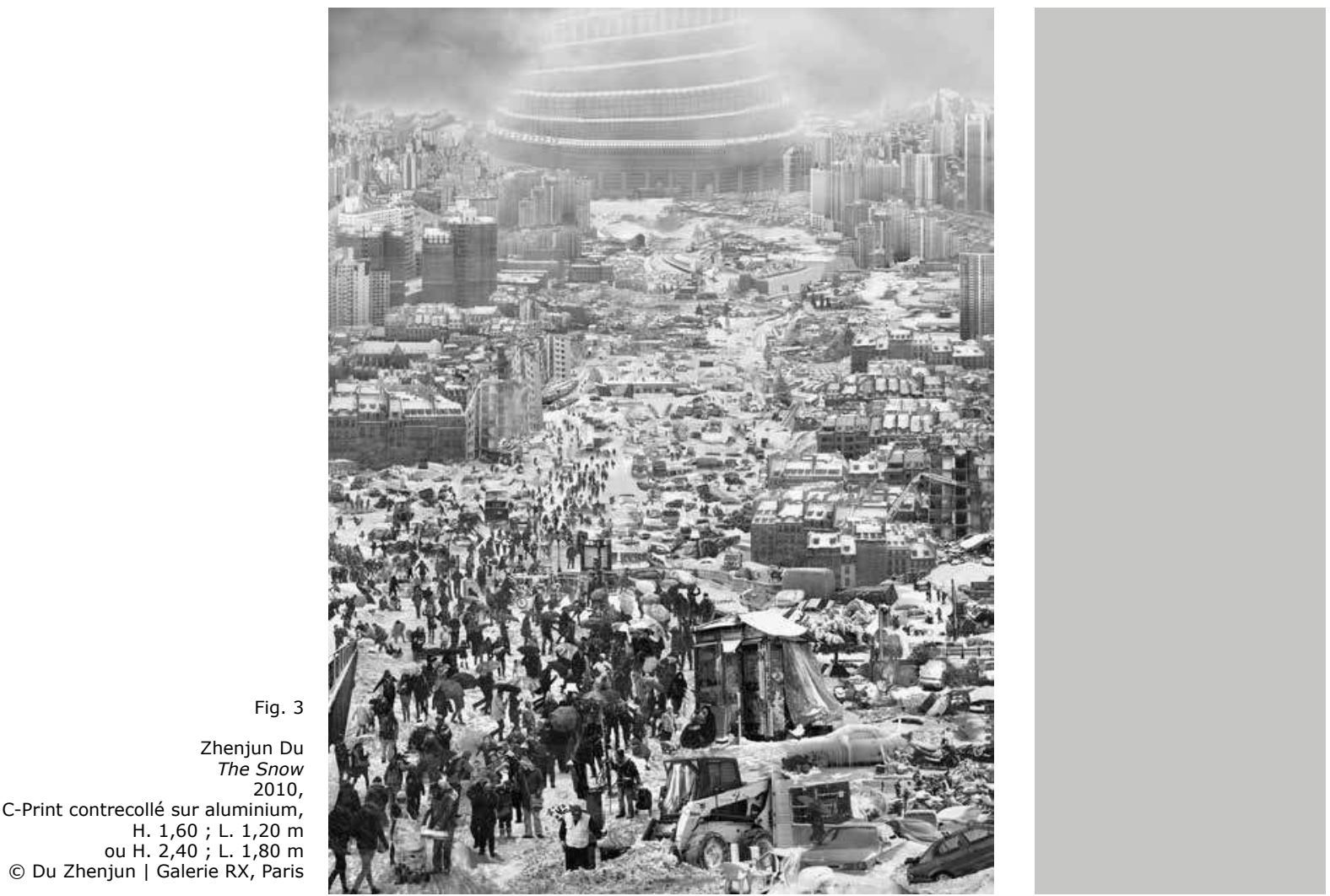

Les attentats du 11 septembre 2001 aux États-Unis semblent avoir suscité une vague de représentations nouvelles de Babel car celles-ci sont, après cette date, relativement nombreuses. L'effondrement des Twin Towers, dont l'image a été maintes fois reprise dans les médias, permet en effet d'établir un parallèle avec la destruction de Babel. Toutefois, il est rare que les représentations de la tour de Babel datant d'après les attentats évoquent directement ou même allusivement la destruction des tours jumelles. Quelques exceptions existent, comme le montage photographique The Accident de Zhenjun Du $\left(2010^{25}\right)$ dans lequel la tour de Babel est prise pour cible par des avions et le Portrait de Jérôme Jacobs de Cédric Tanguy, déjà mentionné, où la tour de Babel de Bruegel, représentée deux fois, est également sur le point d'être frappée par des avions. Dans la série Tower par Wolfe von Lenkiewicz (2008), le dessin Be not afraid représente une bête à multiples têtes perchée sur les Twin Towers en feu ${ }^{26}$. Ces évocations directes des attentats du 11 septembre qui mettent en lien les deux tours et la tour de Babel sont néanmoins limitées. En effet, Babel et Babylone font l'objet d'une critique sévère dans la lecture moderne du mythe et les assimiler aux États-Unis paraît délicat, dans un contexte où les pays occidentaux préferent exprimer leur solidarité avec les victimes. Ainsi, si le mythe trouve un écho particulier dans l'actualité et si par conséquent les œuvres relatives à Babel et Babylone se multiplient après 2001, les artistes hésitent encore à associer explicitement Babel et les Twin Towers. 


\section{De nouvelles formes au service d'une nouvelle vision : la Babel de livres}

La dernière grande transformation iconographique de Babel est son assimilation au monde des livres. La première association de Babel avec une bibliothèque, notée par Sylvie Parizet, se trouve dans un poème de Charles Baudelaire, La Voix, où une bibliothèque est qualifiée de "Babel sombre ". Toutefois, l'identification de Babel à une bibliothèque doit certainement beaucoup à la nouvelle de Jorge Luis Borges, La Bibliothèque de Babel ${ }^{27}$ (1941). Le propos qui y est tenu assimile la bibliothèque de Babel à l'univers. Les hommes qui y vivent en recherchent constamment le sens dans les livres qu'elle contient et qui ne proposent, pour la majorité d'entre eux, qu'une suite de mots incompréhensibles. La nouvelle a été illustrée par Érik Desmazières qui, dans une série d'estampes commandée par les Amis du livre contemporain en 1998, en propose une vision personnelle où la bibliothèque de Babel se caractérise par sa dimension labyrinthique et infinie.

Dans les représentations artistiques, l'association de Babel et Babylone avec le monde des livres n'apparaît qu'à la fin du xxe siècle. Si le propos de Jorge Luis Borges n'est pas repris tel quel, nous pensons qu'il demeure la principale source de cette iconographie tout à fait nouvelle. Nous avons déjà mentionné l'œuvre Mésopotamie - la Papesse d'Anselm Kiefer, réalisée entre 1985 et 1989 et qui assimilait la Mésopotamie à une bibliothèque : aucune allusion à la nouvelle de Borges n'y est faite, du moins explicitement, mais le lien entre le monde des livres et celui de Babylone y est clairement établi. Plus récemment, en 2011, l'artiste Brian Dettmer a proposé une Tower of Babble, sculpture en forme de tour spiralée dont le matériau est constitué de livres sculptés ${ }^{28}$. Les mots qui en surgissent au hasard lui confèrent une dimension poétique et toute allusion à la décadence, la chute ou la destruction disparait.
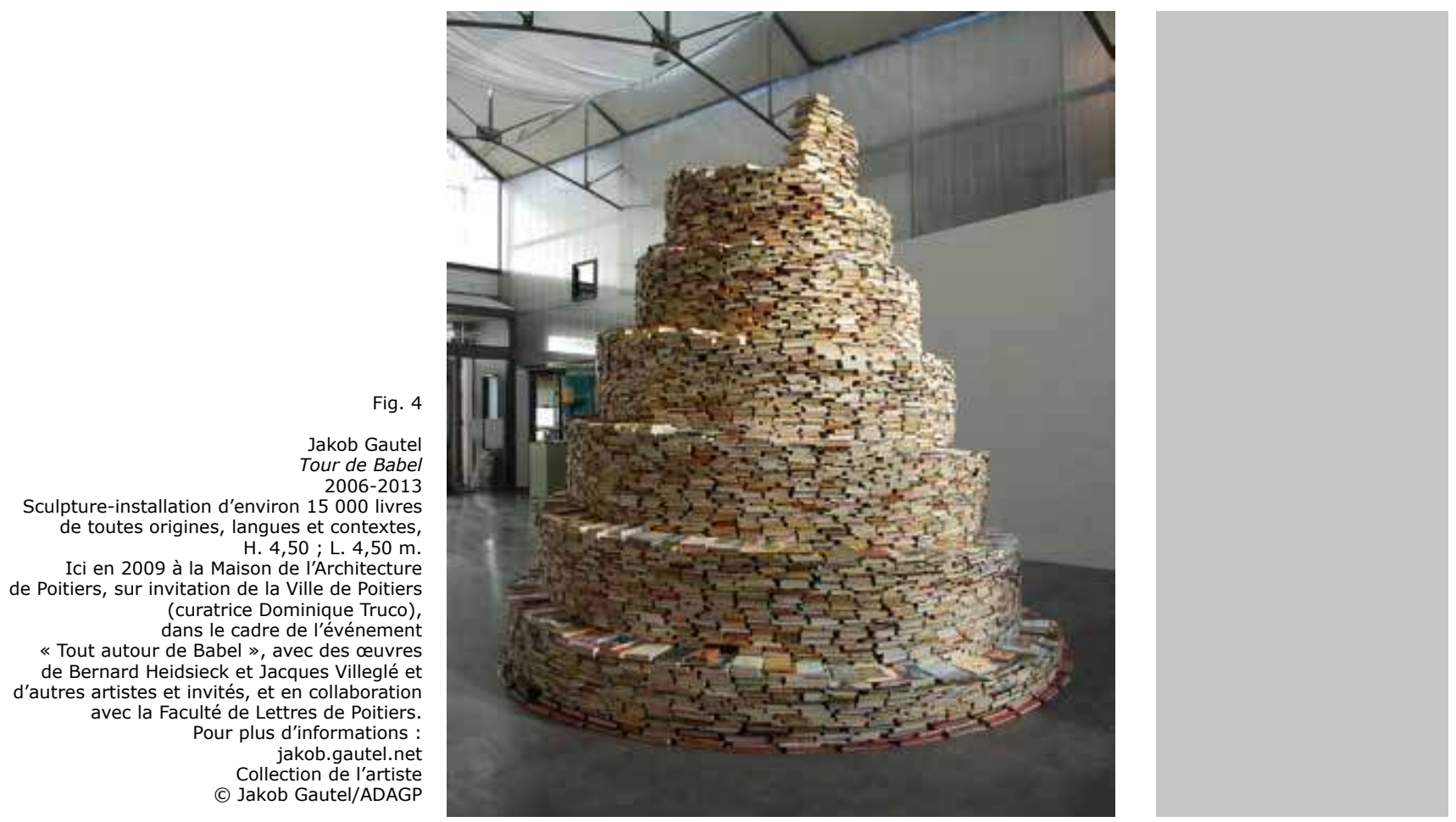

Les tours de livres se développent dans les années 2000 : la Tour de Babel de Jakob Gautel (2006-2013), installation de 15000 livres (fig. 4), se veut une synthèse des productions et de la mémoire de l'humanité, dans ce qu'elle a de meilleur et de plus mauvais. La structure en spirale évoque la croissance

27. Jorge Luis Borges, La Bibliothèque de Babel, dans Fictions, $1^{\text {re }}$ éd. Buenos Aires, Emecé Editores (1956), trad. de l'espagnol par Paul Verdevoye, Ibarra et Roger Caillois, Paris, Gallimard, collection « Folio », 1983

28. Op. cit. note 10, p. $44, n^{\circ} 27$. 
effrénée de cette production tandis que son caractère inachevé invite à faire une pause pour y réfléchir. Autre exemple, La Tour de Babel de 30000 livres élevée par Marta Minujín à Buenos Aires en $2011^{29}$ est une ode à l'union à travers les différences. Comme les œuvres précédemment citées, aucune d'entre elles ne fait explicitement référence à l'œuvre de Borges et, de fait, leur propos est très différent de celui de la nouvelle. Il est cependant clair qu'une iconographie inédite associant Babel au monde des livres semble s'imposer : elle consacre à notre avis une conception nouvelle et plus positive du mythe. En opposition avec la tour-machine menaçante des représentations $\mathrm{du} \mathrm{xx}^{\mathrm{e}}$ siècle, la tour dont le matériau est le livre repose sur un savoir traditionnel et offre la vision d'un progrès plus rassurant que celui proposé par l'industrialisation et les nouvelles technologies. Ainsi, à peine soixante ans après la publication de La Bibliothèque de Babel, l'association de Babel au monde des livres est devenue courante dans les représentations artistiques, enrichissant le mythe par de nouvelles propositions plastiques.

Si le mythe de Babel et de Babylone paraît aujourd'hui avoir un tel succès ${ }^{30}$, c'est qu'il éveille des images et des associations fortes et actuelles. À travers les représentations, il existe une véritable identification des sociétés occidentales contemporaines à la ville-tour, au prix d'une inversion de la signification du mythe. Cette identification, pleinement assumée à partir des années 1920, traduit une mutation profonde des mentalités et des préoccupations qui se fait sur la base d'un rapport difficile et critique des Occidentaux avec leur propre civilisation. Les nouveautés iconographiques mises en place au $\mathrm{xx}^{\mathrm{e}}$ siècle, qu'il s'agisse de la ville-tour pleine de machines, du chantier esclavagiste ou de l'effondrement des tours, semblent s'imposer et emporter l'adhésion. De Paris, nouvelle Babylone, aux attentats du 11 septembre 2001, la culture littéraire et visuelle n'a pas cessé de nourrir et d'entretenir le mythe. De fait, si sa représentation a subi une transformation étonnante, Babel-Babylone n’a jamais paru aussi proche de nous.

\section{L'auteur}

Spécialisée en art et civilisations du Proche-Orient ancien à l'École du Louvre, Ariane Aujoulat est titulaire d'un diplôme de muséologie et d'un diplôme de $2^{\mathrm{e}}$ cycle de l'École du Louvre ainsi que d'un Master 1 Histoire réalisé à l'université de Paris 1 Panthéon - Sorbonne. Son mémoire de recherche Babylone à l'ère de la modernité, dirigé par Dominique Jarrassé et soutenu à l'École du Louvre en 2013 traduit, comme ses précédents travaux, son intérêt pour les approches pluridisciplinaires. 